LA-UR- 98-3466

Approved for public release; distribution is unlimited.

Title:

\section{CONF-980827-}

Author(s):

Submitted to:
J. H. Kamperschroer

J. D. Sherman

T.J. Zaugg

A. H. Arvin

A. S. Bolt

M. C. Richards
LINAC 198

Chicago, IL

August 23-28, 1998

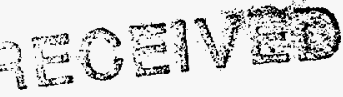

$\operatorname{Ap} 13$ 1993

MASTER
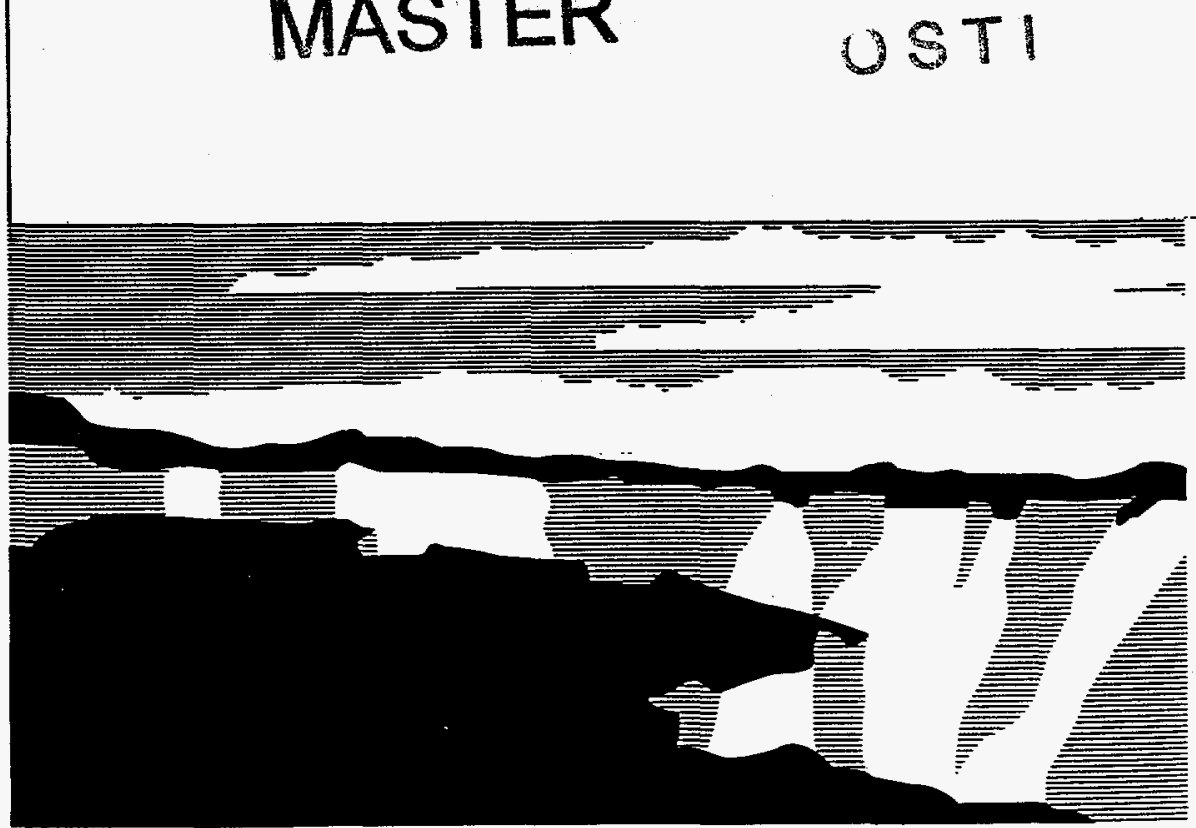

Los Alamos National Laboratory, an affimative actionequal opportunity employer, is operated by the University of Califomia for the

U.S. Department of Energy under contract W-7405-ENG-36. By acceptance of this article, the publisher recognizes that the U.S.

Govemment retains a nonexclusive, royalty-free license to publish or reproduce the published form of this contribution, or to allow others to do so, for U.S. Goverrment purposes. Los Alamos National Laboratory requests that the publisher identify this article as work performed under the auspices of the U.S. Department of Energy. The Los Alamos National Laboratory strongly supports academic freedom and a researcher's right to publish; as an institution, however, the Laboratory does not endorse the viewpoint of a publication or guaranteo its technical correctness. 


\section{DISCLAIMER}

This report was prepared as an account of work sponsored by an agency of the United States Government. Neither the United States Government not any agency thereof, nor any of their employees. makes any warranty, express or implied, or assumes any legal liability or responsibility for the sceuracy, conpleteness, of usefulness of any information, apparntus, produch, of process diselosed, of represents that its use would not infringe privately owned rights. Refereoce herein to any specific commercial product, process, or service by trade asme, tradernart, imanufac. urer, or otherwise does nor necessarily constitute or imply its endorsement, recommendation, of favoring by the United States Government of any agency thereof. The views and opinions of authors expressed herein do not necessarily state or reflect those of the United Stutes Governmeat or any agency thereof. 


\section{DISCLAIMER}

Portions of this document may be illegible in electronic image products. Images are produced from the best available original document. 


\title{
Doppler-shift Proton Fraction Measurement on a CW Proton Injector
}

\author{
J. H. Kamperschroer (General Atomics), \\ J. D. Sherman, T. J. Zaugg (Los Alamos National Laboratory), \\ A. H. Arvin, A. S. Bolt, and M. C. Richards (Savannah River Site)
}

\begin{abstract}
A spectrometer/Optical Multi-channel Analyzer has been used to measure the proton fraction of the $\mathrm{cw}$ proton injector developed for the Accelerator Production of Tritium (APT) and the Low Energy Demonstration Accelerator (LEDA) at Los Alamos. This technique, pioneered by the Lawrence Berkeley National Laboratory (LBNL), was subsequently adopted by the international fusion community as the standard for determining the extracted ion fractions of neutral beam injectors. Proton fractions up to $95 \pm 3 \%$ have been measured on the LEDA injector. These values are in good agreement with results obtained by magnetically sweeping the ion beam, collimated by a slit, across a Faraday cup. Since the velocity distribution of each beam species is measured, it also can be used to determine beam divergence. While divergence has not yet been ascertained due to the wide slit widths in use, non-Gaussian distributions have been observed during operation above the design-matched perveance. An additional feature is that the presence of extracted water ions can be observed. During ion source conditioning at $75 \mathrm{kV}$, an extracted water fraction $>30 \%$ was briefly observed.
\end{abstract}

\section{Introduction}

A diagnostic developed by the magnetic fusion energy community has been successfully utilized on the LEDA injector test facility at Los Alamos[1]. The technique was developed in the late 1970s at LBNL[2] as a means of determining the composition of neutral beams used to heat magnetic fusion plasmas. Extracted $\mathrm{H}^{+}, \mathrm{H}_{2}^{+}$, and $\mathrm{H}_{3}{ }^{+}$interact with background gas to produce fast, excited hydrogen atoms with energies of $E, E / 2$, and $E / 3$, where $E$ is the energy of the extracted ions. By observing the beam at an angle relative to the direction of propagation, the Doppler-shift separates the light from the three species. The wavelength of the Doppler-shifted light is $\lambda=\lambda_{0}(1-\beta \cos \theta)$, where $\lambda_{0}$ is the unshifted wavelength, $\beta=v / c$, and $\theta$ is the angle between the viewing line of sight and the beam's direction of propagation. Beam composition is determined from the quantity of light associated with each Doppler-shifted line. Cross sections for Balmer- $\alpha(\mathrm{H} \alpha)$ production from $\mathrm{H}^{+}, \mathrm{H}_{2}^{+}$, and $\mathrm{H}_{3}{ }^{+}$incident on hydrogen gas have been measured[3], permitting a quantitative measure of the beam composition. In addition to the Doppler shift due to the differing $\beta$ 's, the light from each species is broadened due to the $\cos \theta$ term. For small beam divergence, and neglecting the broadening of the instrument, the line shape is a direct measure of the velocity distribution. In principle, a quantitative measure of the divergence can be made[4]. Collisions of extracted water ions with the background gas produce excited hydrogen atoms with energy $E / 18$, where $E$ is the extraction potential. Water contamination becomes apparent at a level of $<1 \%$

Experiments carried out at Los Alamos have been a successful proof-of-principle test on a proton injector, even though photon fluences from the injector are orders of magnitude below that of large fusion ion sources. The photon production rate is proportional to the beam current, the background gas density, and the H $\alpha$ production cross sections. While the current densities of fusion and APT ion sources are similar, fusion ion sources have much larger extraction areas. Fusion ion source currents are of order $100 \mathrm{~A}$ versus $100 \mathrm{~mA}$ for the LEDA/APT injector. Another significant difference is the background gas density. In neutral beam injectors, the goal is to convert the extracted ions into neutrals by collisions with background gas. For a proton injector, it is important to avoid proton loss via charge exchange to $\mathrm{H}^{0}$. Near the ion source, the background gas density is $\sim 10$ times lower than in a fusion neutral beam injector. The net result is that the $\mathrm{H} \alpha$ production rate in a proton injector is $\sim 10^{4}$ times lower than in a fusion neutral beam injector.

Several factors favor the proton injector. The APT ion source is cw compared to pulse-lengths of a few seconds for fusion ion sources, long integrations are therefore possible. Another advantage is that the neutralization and dissociation of the extracted ion beam, that takes place in the neutral beam injector before the beam reaches the observation point, occurs to a much smaller degree in a proton injector. The problem created by changing the state of the beam is that the H $\alpha$ production cross sections are different for the daughters than for their parents. Therefore, the composition of the beam at the observation point must be known to deduce the composition at the extraction plane. Due to the low gas pressure between the ion source and observation point in a proton injector, a beam composed of $\mathrm{H}^{+}, \mathrm{H}_{2}^{+}$, and $\mathrm{H}_{3}{ }^{+}$can be assumed.

Initial data collection occurred at $50 \mathrm{kV}$, during experiments supporting a $1.25 \mathrm{MeV} \mathrm{cw}$ radio-frequency quadrupole (RFQ)[5]. The RFQ for LEDA and APT has been designed to accept a $75 \mathrm{keV}$ proton beam. A small amount of data was collected after the ion source was regapped for operation at $75 \mathrm{keV}$. 


\section{Description of the Hardware}

A complete Doppler-shift spectroscopy system[6] was borrowed from the Princeton Plasma Physics Laboratory (PPPL). It consists of a $135-\mathrm{mm}$ Canon lens to focus collimated light onto a $600-\mu \mathrm{m}$ glass fiber; an Instruments S.A. 640-mm Czerny-Turner spectrometer; a Princeton Applied Research Corp. vidicon detector; a detector controller; and a computer control console.

The beam was observed $34 \mathrm{~cm}$ downstream from the extractor as it exits the cone that follows the accelerator. The $7-\mathrm{cm}$ exit diameter of the cone equals the $7-\mathrm{cm}$ diameter of the light collection cylinder. At this location the 4-rms beam diameter is $2.2 \mathrm{~cm}$. Since light is collected from the entire horizontal extent of the beam, all of $x$ phase space is observed.

Each pixel in the $500 \times 500$ square detector is $50 \mu \mathrm{m}$. When integrating light from a $43-\mathrm{cm}$ tall PPPL neutral beam injector, the slits of the spectrometer were set at 3 $\mu \mathrm{m}$. In the present case, they were opened to $50 \mu \mathrm{m}$, providing additional light. Opening the slits beyond the $50-\mu \mathrm{m}$ size of the pixels caused broadening of the lines and merging of the two molecular lines. $50 \mu \mathrm{m}$ is a compromise between signal and resolution.

\section{Data and Analysis}

Figure 1 is a spectrum obtained at $50 \mathrm{kV}$ with 120 $\mathrm{mA}$ of ions measured at the first DC current toroid. (All quoted beam currents were measured with this toroid.) The viewing angle, $\theta$, was $60^{\circ}$, the entrance slit of the spectrometer was $50 \mu \mathrm{m}$, and light was integrated for 16 s. Since the beam came towards the line of sight, the Doppler shift was in the blue direction. Left to right, the first three lines are the Doppler-shifted lines from incident $\mathrm{H}^{+}, \mathrm{H}_{2}{ }^{+}$, and $\mathrm{H}_{3}{ }^{+}$. The rightmost, and tallest, line is unshifted $H \alpha(656.2 \mathrm{~nm})$.

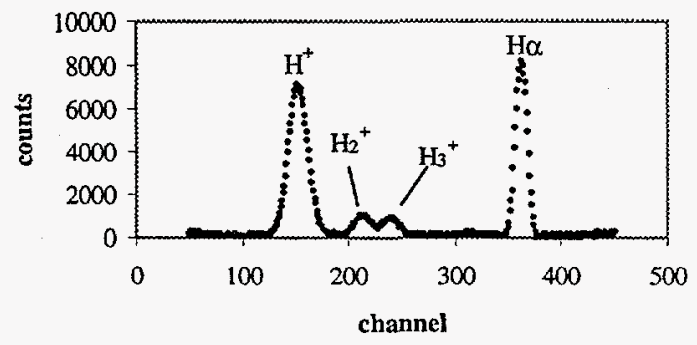

Figure 1. Spectrum at $50 \mathrm{kV}$ and $120 \mathrm{~mA}$ with $50 \mathrm{~mm}$ slits.

Light falls on a portion of the height of the detector corresponding to the diameter of the fiber. Each channel was defined to be one pixel wide by 60 pixels tall (sufficient to include all pixels illuminated by the fiber). The wavelength corresponding to a particular channel can be determined by assigning $656.2 \mathrm{~nm}$ to channel 362 and using a dispersion of $0.016 \mathrm{~nm} /$ channel. The ordinate represents the number of counts detected in each channel.

Proton fractions were obtained by fitting the three Doppler-shifted lines to Gaussians. Error bars of $\pm 3 \%$ for the proton fraction, $17 \%$ for $\mathrm{H}_{2}^{+}$, and $\pm 20 \%$ for $\mathrm{H}_{3}{ }^{+}$are based on uncertainties of $\pm 10 \%$ in calculating the line areas, $\sim \pm 5 \%$ in cross sections[3], and assuming that the gas line density between the ion source and observation point could be as high as $1.5 \times 10^{14} \mathrm{~cm}^{-2}$.

\section{Results}

Proton fractions have been measured for scans of the ion source microwave power and gas throughput. In both cases, the spectrometer and detector were operated in the as in figure 1. Figure 2 shows the results of scanning the $2.45 \mathrm{GHz}$ microwave power to the source over the range of 500 to $1500 \mathrm{~W}$ while the beam energy and gas throughput were fixed at $50 \mathrm{keV}$ and $2.22 \mathrm{sccm}$. Beam current varied from $36 \mathrm{~mA}$ to $140 \mathrm{~mA}$. Due to the wide range over which the beam current changed, the currents to the two electron cyclotron resonance (ECR) solenoids needed to be varied to maintain stable operation.

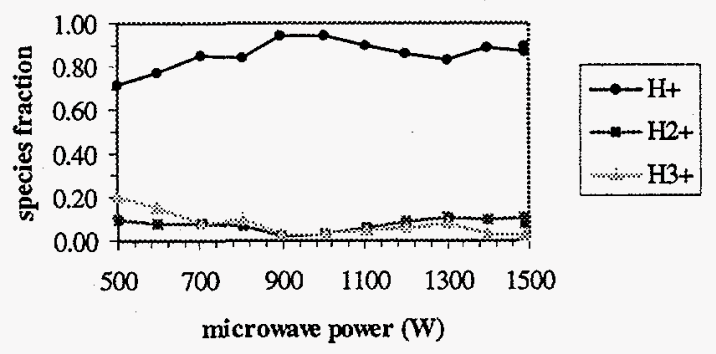

Figure 2. Beam composition as a function of microwave power.

Proton fractions of $0.95 \pm 0.03$ were found at 900 and $1000 \mathrm{~W}$. These values agree with measurements made using an emittance measuring unit modified with a deflection magnet preceding the Faraday cup[1].

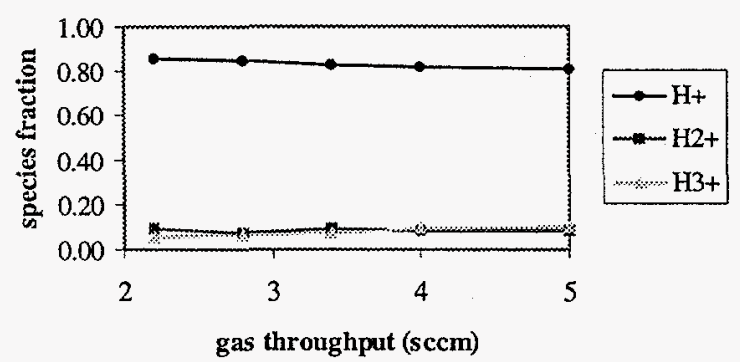

Figure 3. Beam composition as a function of gas throughput.

Figure 3 is the result of a scan where the gas throughput was varied from 2.2 to $5 \mathrm{sccm}$, at a constant microwave power of $1000 \mathrm{~W}$. As the throughput increased 
from 2.2 to $5 \mathrm{sccm}$, beam current decreased from $92 \mathrm{~mA}$ to $71 \mathrm{~mA}$. As above, the currents to the two F.C.R solenoids were adjusted to maintain stable source operation.

It is important to note that the proton fraction corresponding to the $2.2-\mathrm{sccm}$ data point in figure 3 $(85 \%)$ does not agree with the $1000 \mathrm{~W}$ data point in figure 2 (95\%). Source microwave power and throughput were the same in these two cases, but the proton fractions differ by $10 \%$. Inspection of the raw data supports the measured difference. ECR solenoid settings were slightly different. This observation, together with the variations in figure 2 , indicates that the proton fraction is sensitive to minor variations in ion source setpoints.

Figure 4 is a spectrum from $50 \mathrm{kV}, 132 \mathrm{~mA}$ source operation. The source is operating above its designmatched perveance $(110 \mathrm{~mA}$ at $50 \mathrm{kV})$. An important difference between the way this spectrum was acquired and all of those discussed so far is that the slit width was reduced to $20 \mu \mathrm{m}$.

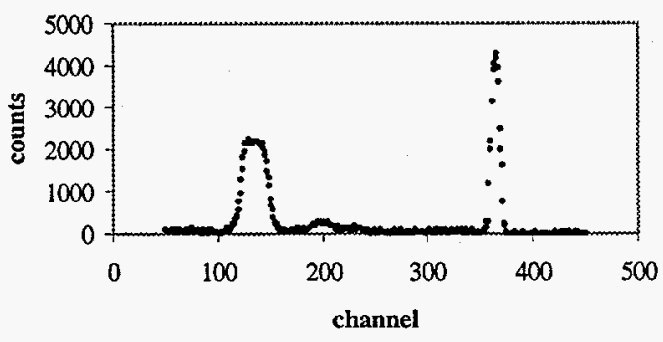

Figure 4. Spectrum taken at $132 \mathrm{~mA}$ with 20 $\mu \mathrm{m}$ slit width showing a nongaussian proton peak.

Note the non-Gaussian shape of the proton line. Such a distribution is indicative of poor beam optics. In this case, it can be attributed to overdense beam extraction. Other data taken with $20-\mu \mathrm{m}$ slits show a return to Gaussian shape as the current was reduced to $110 \mathrm{~mA}$. The data taken with $50-\mu \mathrm{m}$ slits is more Gaussian because the natural line width is convolved with a wider instrument function.

Data were also taken during several hours of ion source operation at $75 \mathrm{keV}$. Immediately prior to when this data was taken, the ion source was exposed to air for accelerator maintenance. The spectrometer slit width was back to $50 \mu \mathrm{m}$.

An interesting spectrum was noted and is shown in figure 5. Situated at channel 300 is a line due to hydrogen atoms extracted as water ions. The presence of significant water in the beam is attributed to the fact that the ion source was just beginning to be conditioned and that water became absorbed on the source walls during its exposure to air.
Cross sections for the production of $\mathrm{H} \alpha$ from water incident on hydrogen have not heen measured. To estimate the water fraction, the required cross sections were estimated from cross sections for the production of $\mathrm{H} \alpha$ for hydrogen atoms incident on water[7].

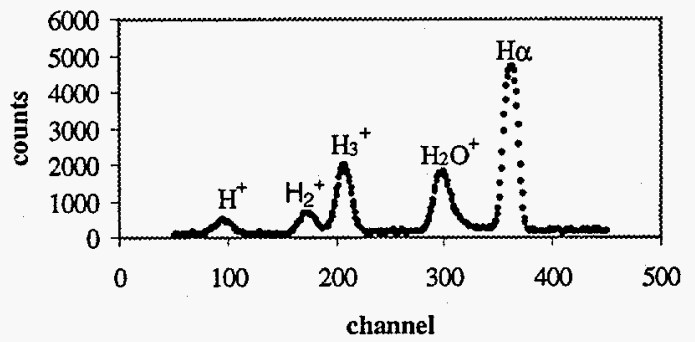

Figure 5. Spectrum at $75 \mathrm{kV}$, just after the ion source was open to air.

The proton fraction is estimated to be in the range of 30 to $40 \%$ while water is 40 to $25 \%$, respectively. There is a large uncertainty in the beam fractions in this case due to the facts that protons are no longer the dominant line and that estimates of the water cross sections differ by a factor of two.

After approximately 10 minutes of $\mathrm{cw}$ operation, the water level in the beam decreased by over an order of magnitude. However, the proton fraction was still poor due to the low source power. Residual gas analysis of the injector vacuum always indicates the presence of water contamination for several days after exposure to air. Water in the ion source cleans up at a more rapid rate due to its active removal by the plasma and the beam.

\section{Acknowledgments}

Funding for this work has been provided by the US Department of Energy. We would also like to thank the neutral beam operations group at the Princeton Plasma Physics Laboratory for the loan of the Optical Multichannel Analysis system.

\section{References}

[1]J. Sherman et al., Rev. Sci. Instrum. 69, 1003 (1998).

[2] C. F. Burrell, W. S. Cooper, R. R. Smith, and W. F. Steele, Rev. Sci. Instrum. 51, 1451 (1980).

[3]I. D. Williams, J. Geddes, and H. B. Gilbody, J. Phys. B 15, 1377 (1982).

[4] G. Bracco, C. Breton, C. de Michelis, M. Mattioli, and J. Ramette, J. Opt. Soc. Am. 71, 1318 (1981).

[5]B. G. Chidley, F. P. Adams, G. E. McMichael, T. T. Ngoc, and T. S. Bhatia, in Proceedings of the 1990 Linear Accelerator Conference.

[6] J. H. Kamperschroer et al., Rev. Sci. Instrum. 58, 1362 (1987).

[7]F. B. Yousif, J. Geddes, and H. B. Gilbody, J. Phys. B. 19, 217 (1986). 